\title{
Study on the mechanism of ErtongKe granules in the treatment of cough using network pharmacology and molecular docking technology
}

\author{
Yu-Long Chen ${ }^{1}$, Wei-Xia Li ${ }^{1,2,3}$, Hui Zhang ${ }^{2}$, Xiao-Yan Wang ${ }^{2,3}$, Shu-Qi Zhang ${ }^{2}$, Ming-Liang Zhang ${ }^{2}$, \\ Jun Han ${ }^{4}$, Kun Li ${ }^{1}$, Ke-Ran Feng ${ }^{1}$, Xiao-Fei Chen ${ }^{2}$, Jin-Fa Tang ${ }^{1,2,3}$ \\ ${ }^{1}$ School of Pharmacy, Henan University of Chinese Medicine, Zhengzhou, China; ${ }^{2}$ Henan Provincial Key Laboratory for Clinical Pharmacy of \\ Traditional Chinese Medicine, Henan Engineering Laboratory of Traditional Chinese Medicine Clinical Evaluation Technology, Pharmaceutical \\ Department, The First Affiliated Hospital of Henan University of Chinese Medicine, Zhengzhou, China; ${ }^{3}$ Collaborative Innovation Center of \\ Traditional Chinese Medicine Prevention and Treatment of Respiratory Diseases, Henan University of Chinese Medicine, Zhengzhou, China; \\ ${ }^{4}$ Yangtze River Pharmaceutical Group, Taizhou, China \\ Contributions: (I) Conception and design: YL Chen, WX Li; (II) Administrative support: JF Tang; (III) Provision of study materials or patients: J Han; \\ (IV) Collection and assembly of data: H Zhang, XY Wang; (V) Data analysis and interpretation: SQ Zhang, ML Zhang; (VI) Manuscript writing: All \\ authors; (VII) Final approval of manuscript: All authors. \\ Correspondence to: Wei-Xia Li. The First Affiliated Hospital of Henan University of Chinese Medicine, Renmin Road No. 19, Zhengzhou 450000, \\ China. Email: liweixia01@126.com; Jin-Fa Tang. The First Affiliated Hospital of Henan University of Chinese Medicine, Renmin Road No. 19, \\ Zhengzhou 450000, China. Email: a0519@163.com.
}

Background: The etiology and pathogenesis of cough are complex. As a Chinese patent medicine that has been on the market, ErtongKe (ETK) granules have a good effect in treating acute and chronic cough in children. The purpose of this research was to determine the bioactive components and possible action mechanisms of ETK in the treatment of cough using an integrated network pharmacology method.

Methods: The Traditional Chinese Medicine Systems Pharmacology (TCMSP) and Swiss target prediction databases were used to screen the potential components and associated targets of ETK. The Genecards database was then used to gather targets interacting with cough. An analysis of the signaling pathways associated with ETK for cough treatment was carried out using the Kyoto Encyclopedia of Genes and Genomes (KEGG) pathway and Gene Ontology (GO) enrichment analysis methods. Cytoscape 3.8.1 was used to design the protein-protein interaction (PPI) and compound-target-pathway networks. Finally, the important genes and active components of ETK were confirmed using Auto Dock vina and Discovery studio software.

Results: Total 242 active components of ETK were screened, 1,173 potential targets related to the ingredients and 4,400 targets related to cough were collected separately. Moreover, 600 candidate targets and 39 signaling pathways were determined. We also screened out the following core components, including tuberostemonone, quercetin, kaempferol, praeruptorin E, stigmasterol, oroxylin A, and other potentially active ingredients. At the same time, 8 core targets, including $\mathcal{F} U N$, PIK3CA, PIK3R1, MAPK14, EGFR, SRC, $A K T 1$, and $M A P K 1$, and 20 key pathways, including the cAMP signaling pathway, calcium signaling pathway, and PI3K-Akt signaling pathway among others, were also selected. All the 8 core targets were verified by molecular docking.

Conclusions: This research established that ETK exerts anti-cough activity by modulating several targets and pathways through multiple components. Additionally, the pooled results shed light on ETK compounds being investigated as potential antitussives.

Keywords: ErtongKe granules; cough; network pharmacology; molecular docking; action mechanism 
Submitted Jul 29, 2021. Accepted for publication Nov 12, 2021.

doi: 10.21037/apm-21-2807

View this article at: https://dx.doi.org/10.21037/apm-21-2807

\section{Introduction}

The etiology and pathogenesis of cough are complex. Cough is prevalent in childhood and is one of the most common causes of pediatric consultation on a daily basis (1). The reasons are distinct from those seen in adults, and pediatric standards must be followed to ensure proper diagnosis and treatment. While viral infection is the most frequent cause of cough in children, all children with a persistent cough, defined as a cough lasting longer than 4-8 weeks or "chronic cough", must be thoroughly examined to rule out specific reasons that span the pediatric pulmonology spectrum. Cough therapy should be determined by the cause. Around $80 \%$ of cases can be identified optimally, and $90 \%$ of them can be treated well. Empirical therapy based on cough features may be beneficial in certain instances of "nonspecific chronic cough" in which no underlying disease can be identified. Chinese medicine (CM), with its emphasis on systemic functional changes, is better suited to diseases with a complicated etiology in this respect.

The prescription of ErtongKe (ETK) granules is derived from Jiegeng Decoction in Eastern Han Dynasty' medical classic ShangHanLun, San'ao Decoction in Song Dynasty' TaiPing HuiMin HefifuFang, and Zhisou Powder in Qing Dynasty' YiXueXinWu. It is composed of 9 traditional Chinese herbs, including Aster Tataricus (Ziwan, ZW), Radix Stemonae (Baibu, BB), Eriobotryae Folium (Pipaye, PPY), Armeniacae Semen Amarum (Kuxingren, KXR), Peucedanum Praeruptorum (Qianhu, QH), Ephedrae Herba (Mahuang, MH), Platycodon Grandiflorus (Jiegeng, JG), Polygoni Tinctorii Folium (Liaodaqingye, LDQY), and Licorice Root (Gancao, GC). ETK is a one of the commercial medicinal products approved by the China Food and Drug Administration, which has more than 10 years of clinical application experience. And it is an experience prescription in pediatrics and its medicinal nature is peaceful, reasonable prescription, proper compatibility, more suitable for children's physiological characteristics. Nevertheless, there are few literatures on the material basis and action mechanism of ETK. In the existing literatures, only the content of ephedrine hydrochloride in ETK has been determined (2). The complexity components and the compatible application of many CMs constitutes their diversity efficacies. The interpretation of the material basis and action mechanism of CMs is an effective method to promote the rational use of CMs. However, the traditional method has the disadvantages of long time and requiring a lot of human and financial resources. The system integration network pharmacology developed in recent years has the advantages of rapidity and simplicity, which is a practical approach for screening the active components and pharmacological effects of herbs or Chinese herbal formulas. In order to promote the clinical rational use of ETK, it is necessary to systematically study its pharmacodynamic substances and action mechanism. Therefore, in this research, a component-targetpathway network was constructed to explore its possible mechanisms on cough. Figure 1 depicts a detailed flowchart of the research design process. Since most of the current literatures on network pharmacology is based on database to screen the compounds of CMs, lacking of the information supplement from literatures on pharmacokinetics and pharmacology. First of all, the potential components and cough intersection genes were obtained through screening databases and literatures, so as to more comprehensively and accurately reflect the active substances and action targets of ETK. Subsequently, the protein-protein interaction (PPI) and herbs-ingredients-targets-pathways network were built. Additionally, we analyzed the Gene Ontology (GO) and the Kyoto Encyclopedia of Genes and Genomes (KEGG) databases. Finally, the molecular docking simulation was used to verify the putative bioactive components and key targets. It can provide a reference for the in-depth study of pharmacodynamic substances and action mechanism of ETK.

We present the following article in accordance with the STREGA reporting checklist (available at https://dx.doi. org/10.21037/apm-21-2807).

\section{Methods}

\section{Collection of active ingredients and associated targets}

The Traditional Chinese Medicine Systems Pharmacology (TCMSP, https://www.tcmsp-e.com/) database contains a large number of compounds derived from herbal medicines, as well as their associated protein targets and pharmacokinetic characteristics (3). It was used to collect putative ETK active ingredients by filtering with oral bioavailability (OB) 


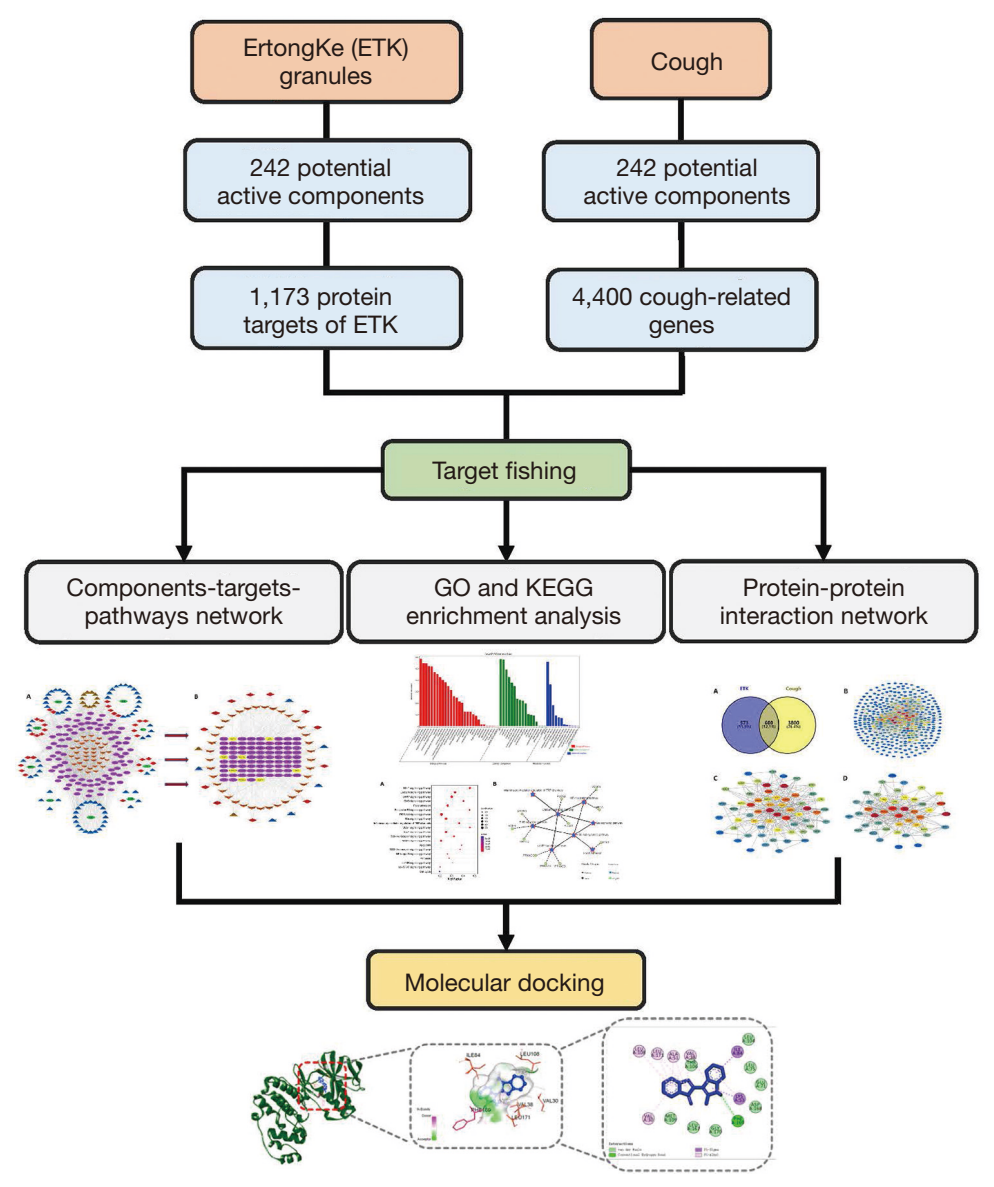

Figure 1 The detailed flowchart of the study design. GO, Gene Ontology; KEGG, Kyoto Encyclopedia of Genes and Genomes.

of $30 \%$ and drug-likeness (DL) of 0.18 (4). In addition, in order to avoid the omission of the main components and pharmacologically active components in each CM by the screening conditions of TCMSP database, in the early stage, we searched the main pharmacodynamic components of 9 herbs in ETK through the PubMed literature database to supplement and finally confirmed 242 active components. Following that, the TCMSP and Swiss Target Prediction (http://www.swisstargetprediction.ch/) databases were used to predict protein targets interacting with these probable active components (5).

\section{Acquisition of disease-associated targets and potential genes}

The information on cough-associated target genes was obtained using the GeneCards database (https://www. genecards.org/), which is a comprehensive encyclopedia on proteomics, genomics, and transcriptomics functions (6). The terms "cough" and "bronchitis" were used to screen for targets related to coughing. To minimize false positives, we included only the selected targets that were closely related to the disease and excluded repeated targets. Following that, disease-related and component-predicted expression target protein names were imported into the Uniprot database (https://www.uniprot.org/) and protein names were optimized. Finally, we created a Venn diagram out of the 2 sets of genes we acquired and evaluated the genes that intersected as possible candidates for further investigation. The study was conducted in accordance with the Declaration of Helsinki (as revised in 2013).

\section{Creating a PPI network}

The PPI network was created by importing candidate genes into the STRING database (https://string-db.org/) and 
setting the confidence level to 0.95 (7). The PPI network was shown using the Cytoscape program v3.8.1 (8). Then, 3 topological characteristics were computed to identify the essential genes: "Degree", "Betweenness", and "Closeness". The "Degree" parameter indicates the number of edges connected with a node, the term "Betweenness" refers to the number of shortest routes connecting 2 nodes, while "Closeness" denotes the inverse of the number of distances.

\section{GO and KEGG enrichment analysis}

To further investigate the roles of the aforementioned screening targets in biological signaling pathways, we imported the intersecting genes into DAVID Bioinformatics Resources 6.8 (https://david.ncifcrf.gov/) for GO and KEGG analysis. The DAVID database is a web-based resource for understanding the molecular processes and signaling mechanisms behind possible targets for cough therapy (9). After that, we chose the identifier to be used as the official gene symbol, set the list type to Gene List, and limited the species to Homo sapiens.

\section{Creation of a berb-ingredient-target-patbway network}

We built a herb-ingredient-target-pathway network using Cytoscape software to improve our possible active components as well as critical genes that ETK potentially targets to treat cough (8). The nodes in the network representing hrebs, components, disease, targets, and pathways were represented by various shapes and colors.

\section{Molecular docking analysis of ingredient-target binding activity}

We were able to confirm the binding activity of the main components of ETK with their corresponding potential targets that may be expressed in the context of disease by applying Auto Dock vina (version 1.1.2) (10) for molecular docking. The component's two-dimensional structure was retrieved from the PubChem database and then converted to MOL2 format using ChemDraw software. Additionally, the main target protein was retrieved from the Protein Data Bank (PDB, https://www.rcsb.org/). The following criteria were used to screen for target proteins: (I) organisms had to be Homo sapiens; (II) resolution had to be 3.0A; (III) the release date had to be as late as feasible; and (IV) priority was given to those with novel ligands. AutoDockTools was used to process the components and protein structures (version 1.5.6). Discovery studio software was used to display the combinations of the most excellent docking scores (version 4.5.0).

\section{Results}

\section{Components of ETK and potential genes for cough}

We screened 210 active compounds in the TCMSP database by using parameters of $\mathrm{OB} \geq 30 \%$ with $\mathrm{DL} \geq 0.18$. Additionally, this study collected 32 active components of cough treatment that did not meet the $\mathrm{OB}$ and DL values through a literature search. Meanwhile, 1,173 target genes interacting with these putative components were collected by the Swiss Target Prediction and TCMSP databases. Furthermore, there remained 4,400 cough-associated genes from the GeneCards database. Ultimately, a total of 600 intersecting genes, also known as potential genes, were gathered for the purpose of studying the various processes underlying the action of ETK in the treatment of cough (Figure 2A).

\section{Analyses of the PPI network}

The 600 potential genes were linked to create an initial PPI network with 391 nodes and 1,507 edges, and 209 isolated target genes were eliminated (Figure $2 B$ ). Additionally, the top 2 clusters of PPIs were created (Figure 2C,2D). STAT3, $T P 53$, and $S R C$ were the primary targets for one cluster $(60$ nodes and 302 edges), while HRAS, PIK3CA, and PTK2 were the core targets for another cluster (44 nodes and 230 edges). Based on the study of this network, the crucial targets of ETK for cough were selected as those with higher values of "Degree", "Betweenness", and "Closeness" (all of which were greater than the median value). Besides, the higher the connection degree of the target, the warmer the hue of the node, and vice versa.

\section{Results of GO and KEGG enrichment analysis}

\section{Analyses of GO enrichment}

To observe the biological functions of ETK target genes involved in cough, the DAVID database was used to perform GO analysis. A total of 365 GO entries were acquired based on $\mathrm{P}$-value $(\mathrm{P}<0.05)$ and Gene numbers (Count $\geq 10$ ). They included 235 items of biological process (BP), 66 items of cellular components (CC), and 
A
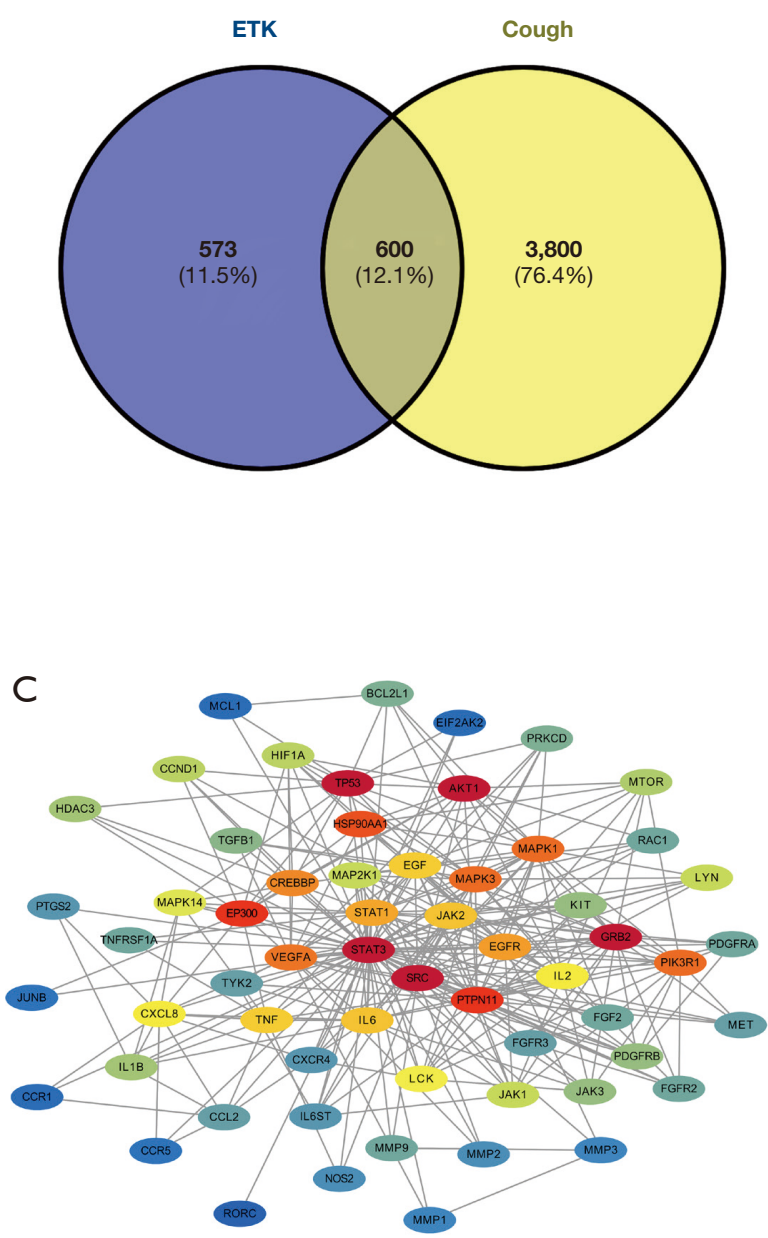

B
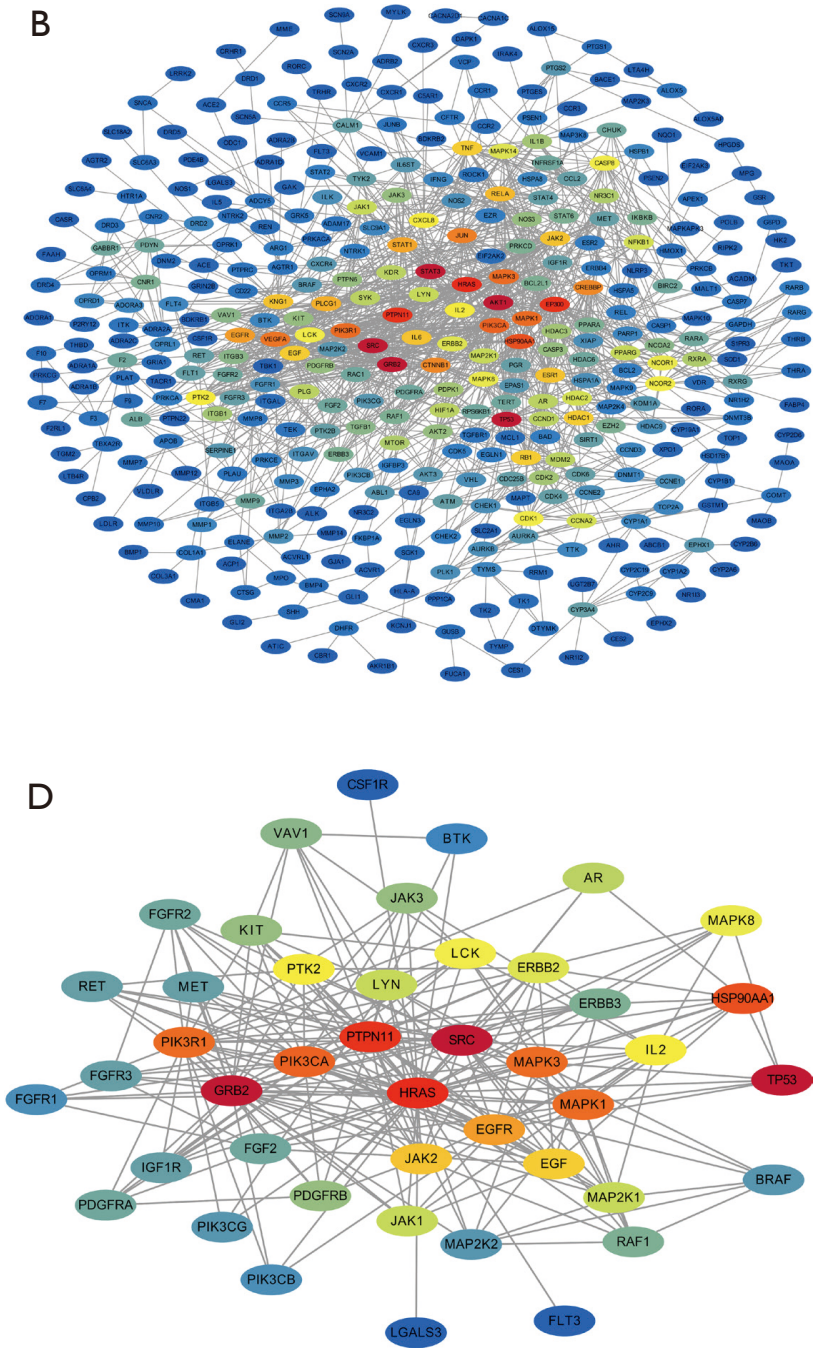

Figure 2 Venn diagram and protein-protein network representational images. (A) The 600 intersection genes; (B) a full protein-protein network; (C) a single protein-protein cluster with 60 nodes and 302 edges; (D) a single protein-protein cluster with 44 nodes and 230 edges.

64 items of molecular function (MF). As illustrated in Figure 3, the markedly enriched BP terms were shown, including cellular processes, biological regulation, response to stimulus, metabolic process, immune system process, and signaling, among others. For CCs, the items with significant enrichment were in the organelle, membrane, synapse, and extracellular region, among others. For MFs, the items with significant enrichment were in drug binding, catalytic activity, molecular transducer activity, transcription regulator activity, and transporter activity, among others.

\section{Analyses of KEGG pathway enrichment}

The KEGG enrichment analysis of ETK for cough $(\mathrm{P}<0.05)$ was also carried out using the DAVID database.
There was a total of 148 pathways discovered. Twenty significant signaling pathways (Figure $4 A$ ) were selected for further analysis, including the calcium signaling pathway (hsa04020), cAMP signaling pathway (hsa04024), focal adhesion (hsa04510), PI3K-Akt signaling pathway (hsa04151), and Ras signaling pathway (hsa04014), among others. Besides that, through searching 20 key signaling pathways, 8 core signaling pathways related to ETK on the cough treatment were found (Figure 4B).

\section{ETK-component-target-patbway network}

After 327 targets in the signaling pathway were re-matched with the intersection targets, 214 active components were 


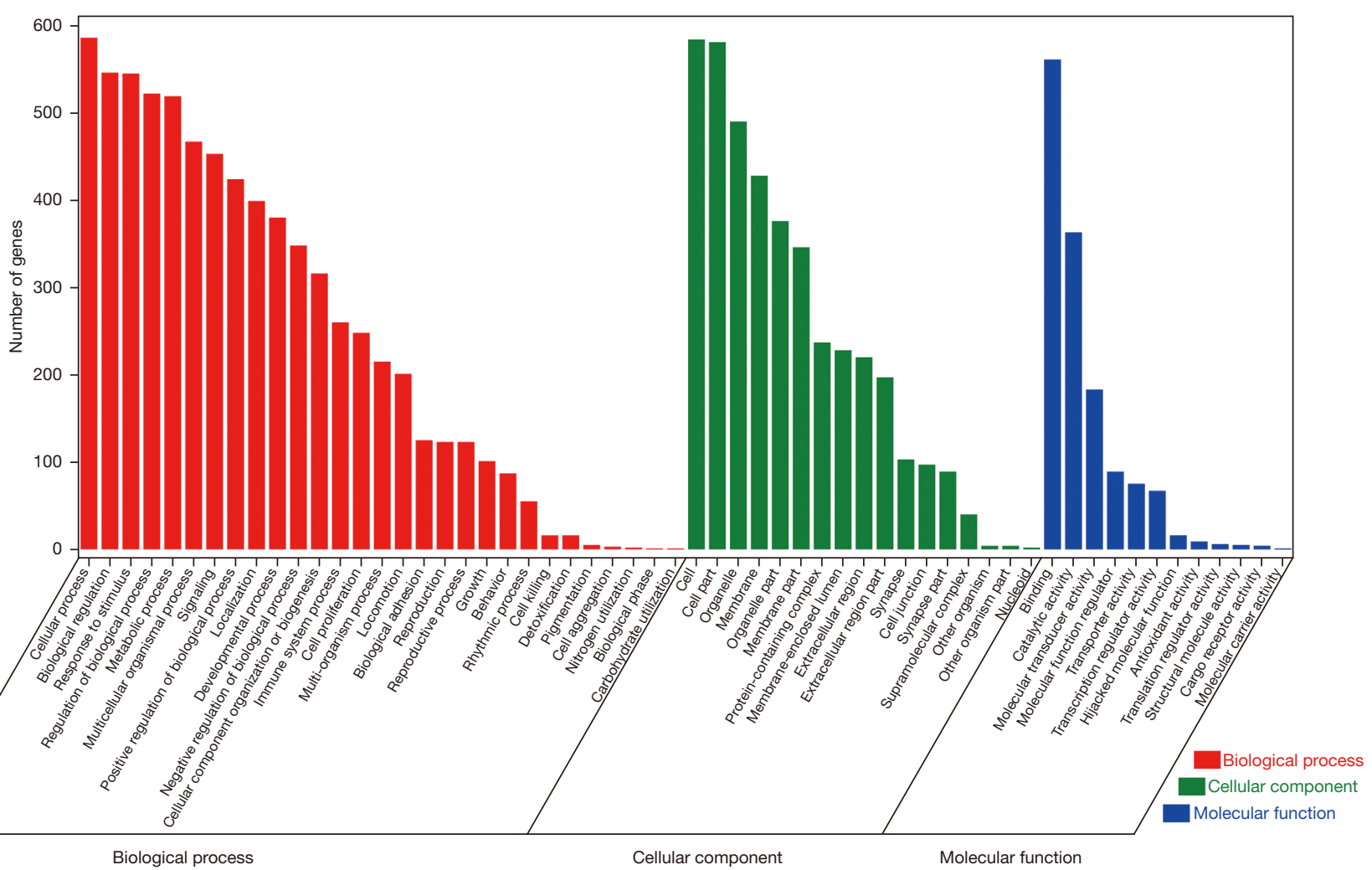

Figure 3 Analyses of the cellular components, biological processes, and molecular functions of the 600 prospective targets. The horizontal axis of the histogram displays the core items of the Gene Ontology enrichment analysis; the vertical axis indicates the degree of enrichment of prospective targets of each item. The greater the number of genes, the more critical this component becomes.

screened. A total of 214 components, 327 targets, and 39 pathways were imported into Cytoscape software, and then the complete "ETK-component-target-pathway" network (Figure 5A) was constructed. Furthermore, according to the network topology analysis, the degree, betweenness centrality, and closeness centrality of each node and other network parameters were obtained to screen out the top 2 core components of each herb in ETK and construct the "core component-target-pathway network" (Figure 5B).

As can be seen in Figure 5B, there were 20 core components, 86 core targets, and 39 core pathways. The core components' primary information is shown in Table 1, among which 10 components, such as platycodin A, protostemonine, norephedrine, and amygdalin, among others, failed to meet the standard levels of $\mathrm{OB}$ and DL values. However, in the literature, these components have been shown to have some pharmacological activity. In addition, the top 30 core targets (Table 2) in the network were selected according to the Degree value.

\section{Molecular docking results and analysis}

According to the analysis in Figure 5 described above, 8 core potential targets and 18 related components were screened out. Then, the binding ability of the protein to its potentially active small molecules was evaluated by the molecular docking method. The molecular docking results showed that the docking scores of the other 15 potentially active ingredients with related targets except for PIK3CA-

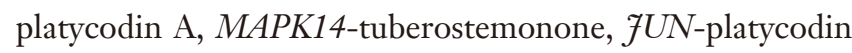
$\mathrm{C}$, and JUN-astersaponin A were all less than -4.0, which indicated that they had potential binding activity. Among them, MAPK14-indirubin, PIK3CA-quercitrin, $A K T 1$-isorhamnetin, MAPK1-shionoside C, EGFRtuberostemonone, SRC-hyperoside, PIK3R1-kaempferol, and $\mathcal{F} U N$-kaempferol had the best binding activity compared with the others (Table 3). And five representative molecular docking results including 3D and 2D images were drawn and displayed (Figure 6). 
A

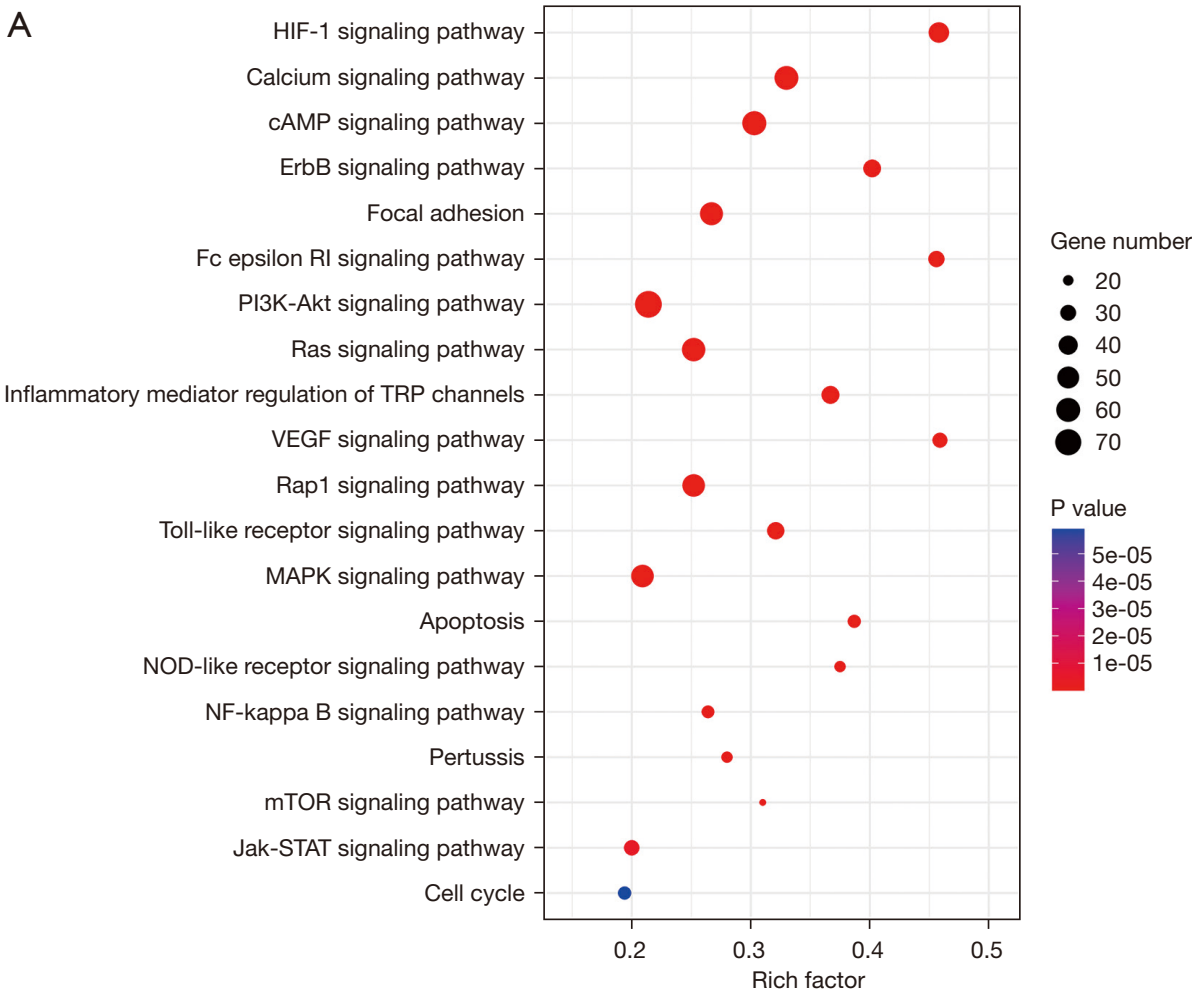

B

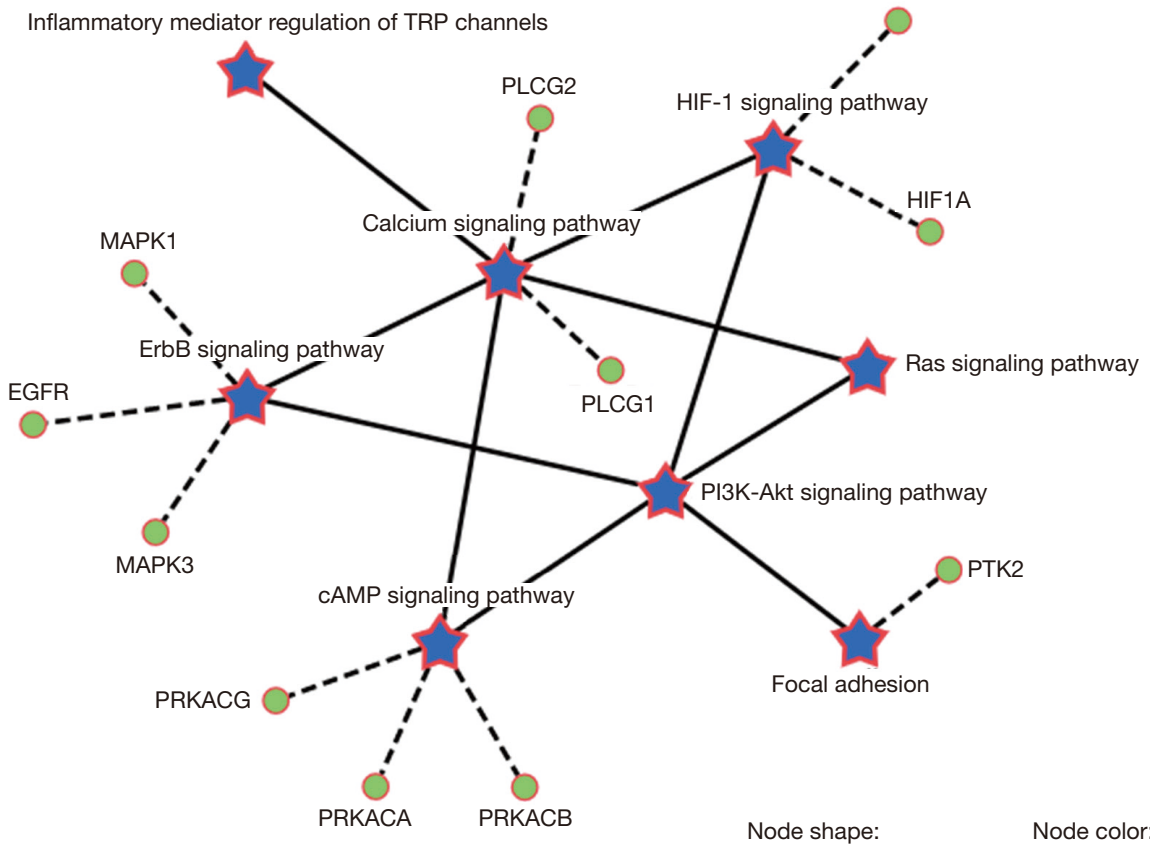

$\star$ Pathway

- Gene
Pathway

Gene

Figure 4 KEGG pathway enrichment analysis results. (A) The KEGG enrichment bubble graphic. The size of the bubble reflects the number of core targets engaged in the phrase, while the color scale from red to blue denotes the $\mathrm{P}$ value from tiny to enormous, with red indicating more importance. (B) A network comprised of 8 critical signaling pathways. KEGG, Kyoto Encyclopedia of Genes and Genomes. 

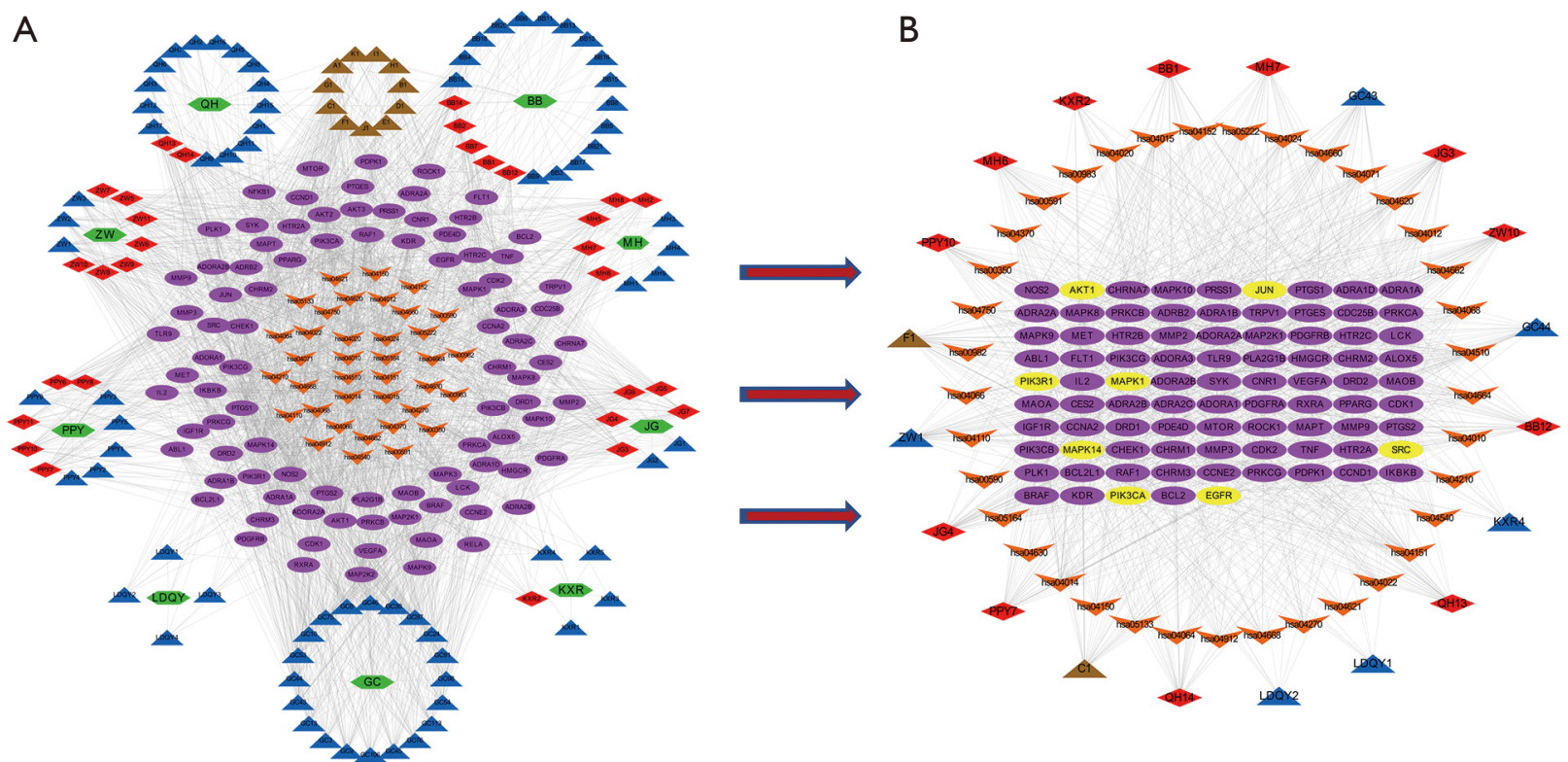

Figure 5 The ErtongKe granules-component-target-pathway network. The green nodes are traditional Chinese medicine. The red nodes are the pharmacologically active ingredients through literature retrieval. The blue nodes are potential active ingredients. The brown nodes represent the typical components of traditional Chinese medicine. The purple nodes are the key targets. The orange nodes are the core pathways. The yellow nodes are the critical targets in the protein-protein interaction network. (A) The complete ErtongKe granulescomponent-target-pathway network; (B) the core component-target-pathway network.

\section{Discussion}

\section{Active ingredients}

In the analysis of the ETK-component-target-pathway network, the active ingredients, including tuberostemonone, quercetin, kaempferol, praeruptorin E, stigmasterol, and oroxylin A, among others, have the potential to relieve cough, dispel phlegm, and have anti-inflammatory and antimicrobial effects.

In mouse models of phenol red secretion, ammoniainduced cough, and xylene-induced ear swelling, the results showed that compounds such as astersaponin $\mathrm{A}$ and shionoside $\mathrm{C}$, which are derived from $\mathrm{ZW}$ have significant expectorant, antitussive, and anti-inflammatory effects (19). The total Stemona alkaloid fractions were evaluated using the in vivo citric acid-induced guinea pig cough model, and the results suggested that they possess antitussive activity (11). Furthermore, protostemonine exhibits anti-inflammatory properties when applied to acute lung damage. According to recent studies, protostemonine alleviates asthmatic inflammation by attenuating $I L-4-$ induced expression of STAT6 phosphorylation, KLF4, and IRF4 in bone marrow-derived macrophages. It might therefore be a potential agent for treating asthma (12). Norephedrine is commonly found in cough or cold remedies. The compound, an alpha-adrenergic receptor agonist, is used to treat colds and relieve nasal congestion and coughing. Its effect is similar to that of ephedrine, which has a contractile effect on skin mucosa and visceral blood vessels (15). Numerous investigations have shown that quercitrin at a certain dose substantially inhibited ear edema caused by xylene and showed mild anti-microbial activity against bacteria strains (17). Hyperoside ameliorated ovalbumin (OVA)-induced allergic airway inflammation by activating the Nrf2 signaling pathway. It substantially reduced the infiltration of inflammatory cells and the levels of $I L-4, I L-5$, $I L-13$, and $I g E$, and prevented OVA-induced oxidative stress, as measured by decreased MDA and increased GSH and SOD levels. Additionally, hyperoside reduced NF- $\kappa \mathrm{B}$ activation caused by lipopolysaccharide (LPS) and activated the Nrf2/HO-1 signaling pathway (18). A naturally occurring cyanogenic chemical called amygdalin has been used to cure a variety of ailments in Asia, Europe, and other parts of the world for centuries (14). Platycodin A and platycodin D inhibited the expression of LPS-induced iNOS, COX-2 protein and mRNA, and prostaglandin E2 
Table 1 ErtongKe granules's main active components in the treatment of cough

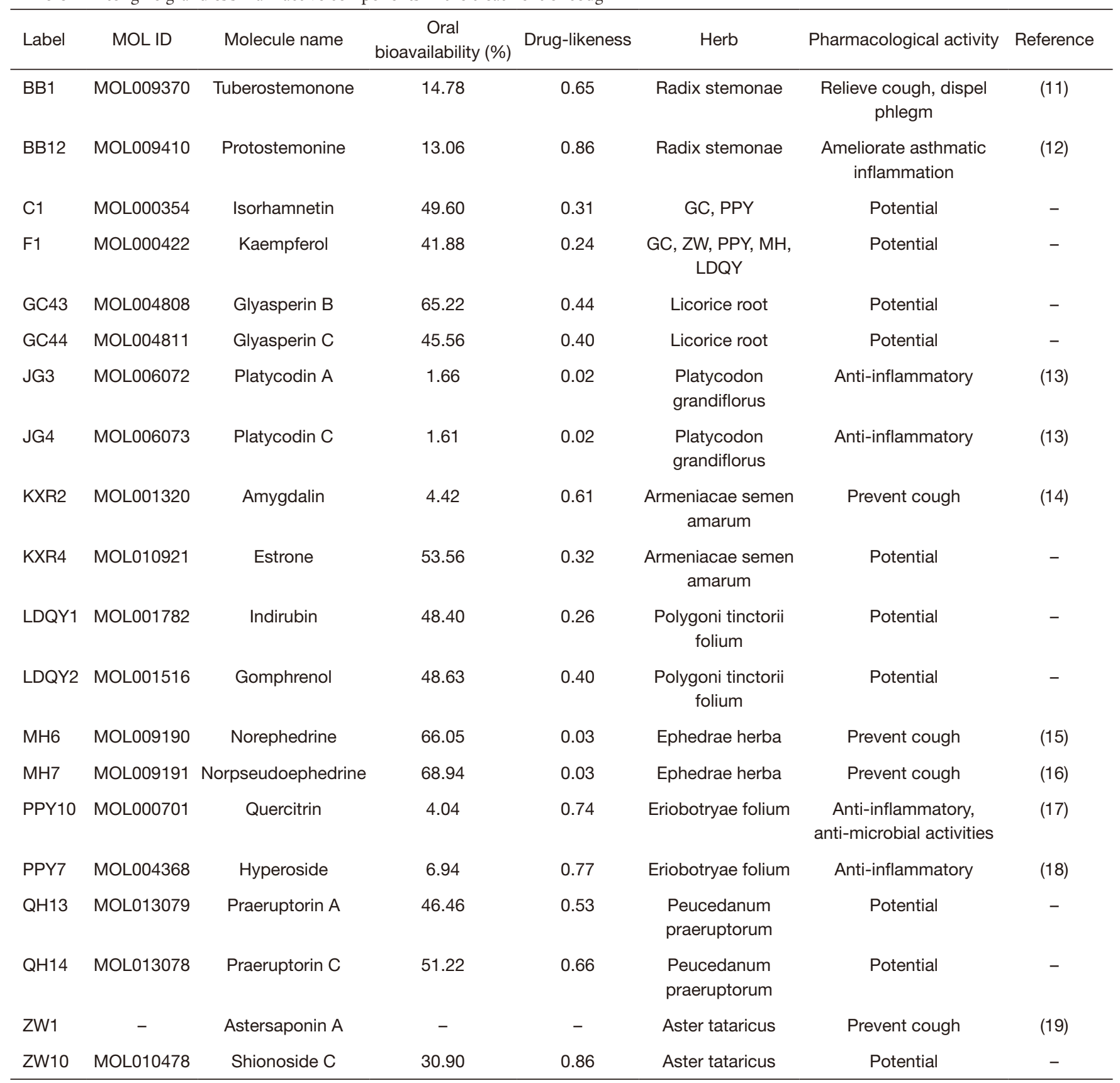

MOL ID represents the ID information defined by the chemical component in the Traditional Chinese Medicine Systems Pharmacology database; $\mathrm{C} 1$ represents one of the common components of Licorice Root and Eriobotryae Folium; F1 represents one of the common components of Licorice Root, Aster Tataricus, Eriobotryae Folium, Ephedrae Herba, and Polygoni Tinctorii Folium; BB, Radix Stemonae; ZW, Aster Tataricus; BB, Radix Stemonae; PPY, Eriobotryae Folium; KXR, Armeniacae Semen Amarum; QH, Peucedanum Praeruptorum; MH, Ephedrae Herba; JG, Platycodon Grandiflorus; LDQY, Polygoni Tinctorii Folium; GC, Licorice Root. 
Table 2 ErtongKe granules' primary targets and topological parameters in cough therapy

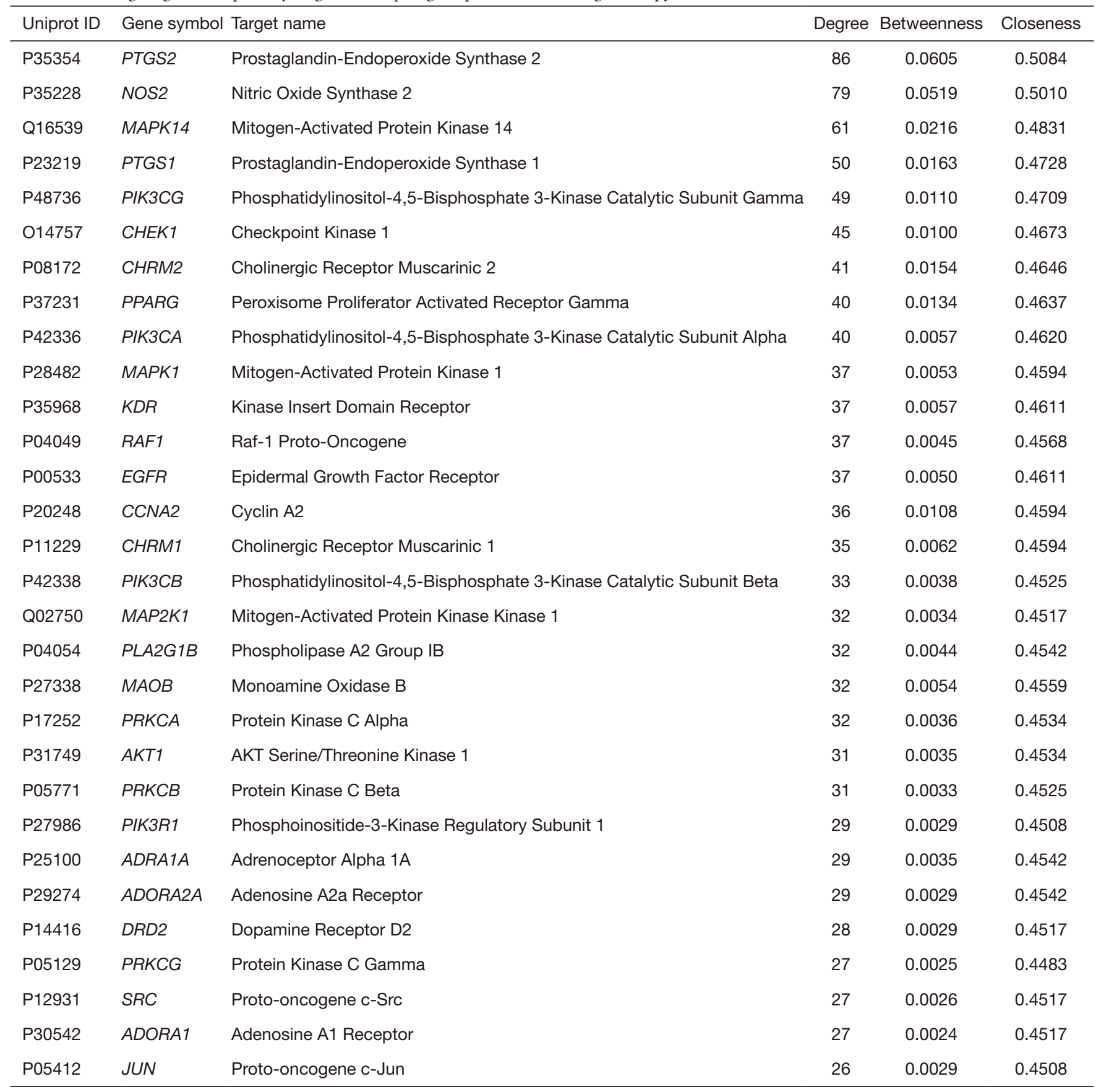

PTGS2, prostaglandin-endoperoxide synthase 2; NOS2, nitric oxide synthase 2; MAPK14, mitogen-activated protein kinase 14; PTGS1, prostaglandin-endoperoxide synthase 1; PIK3CG, phosphatidylinositol-4,5-bisphosphate 3-kinase catalytic subunit gamma; CHEK1, checkpoint kinase 1; CHRM2, cholinergic receptor muscarinic 2; PPARG, peroxisome proliferator activated receptor gamma; PIK3CA, phosphatidylinositol-4,5-bisphosphate 3-kinase catalytic subunit alpha; MAPK1, mitogen-activated protein kinase 1; KDR, kinase insert domain receptor; RAF1, Raf-1 Proto-Oncogene; EGFR, epidermal growth factor receptor; CCNA2, Cyclin A2; CHRM1, cholinergic receptor muscarinic 1; PIK3CB, phosphatidylinositol-4,5-bisphosphate 3-kinase catalytic subunit beta; MAP2K1, mitogen-activated protein kinase kinase 1; PLA2G1B, phospholipase A2 group IB; MAOB, monoamine oxidase B; PRKCA, protein kinase C alpha; AKT1, AKT serine/threonine kinase 1; $P R K C B$, protein kinase $C$ beta; $P I K 3 R 1$, phosphoinositide-3-kinase regulatory subunit 1; $A D R A 1 A$, adrenoceptor alpha $1 \mathrm{~A} ; A D O R A 2 A$, adenosine A2a receptor; $D R D 2$, dopamine receptor D2; PRKCG, protein kinase C gamma; SRC, proto-oncogene c-Src; ADORA1, adenosine A1 receptor; JUN, proto-oncogene c-Jun. 
Table 3 The 8 core targets with their source composition information and docking score

\begin{tabular}{|c|c|c|c|}
\hline No. & Target (PDB-ID) & Molecule name & $\begin{array}{c}\text { Docking score } \\
\text { (kcal/moL) }\end{array}$ \\
\hline \multirow[t]{7}{*}{1} & MAPK14 (5OMG) & Indirubin & -9.0 \\
\hline & & Isorhamnetin & -8.8 \\
\hline & & Kaempferol & -8.5 \\
\hline & & Gomphrenol & -8.2 \\
\hline & & Amygdalin & -7.4 \\
\hline & & Protostemonine & -6.7 \\
\hline & & Shionoside C & -5.3 \\
\hline \multirow[t]{8}{*}{2} & PIK3CA (6AUD) & Quercitrin & -8.7 \\
\hline & & Praeruptorin C & -8.4 \\
\hline & & Glyasperin C & -8.3 \\
\hline & & Shionoside C & -8.3 \\
\hline & & Hyperoside & -7.9 \\
\hline & & Praeruptorin A & -7.5 \\
\hline & & Glyasperin B & -7.3 \\
\hline & & Protostemonine & -6.9 \\
\hline \multirow[t]{5}{*}{3} & AKT1 (6M89) & Isorhamnetin & -7.6 \\
\hline & & Kaempferol & -7.6 \\
\hline & & Tuberostemonone & -7.4 \\
\hline & & Protostemonine & -7.3 \\
\hline & & Hyperoside & -7.1 \\
\hline \multirow[t]{2}{*}{4} & MAPK1 (5OMH) & Shionoside C & -7.9 \\
\hline & & Tuberostemonone & -6.7 \\
\hline \multirow[t]{10}{*}{5} & EGFR (5WB7) & Tuberostemonone & -5.0 \\
\hline & & Hyperoside & -5.0 \\
\hline & & Kaempferol & -4.9 \\
\hline & & Isorhamnetin & -4.9 \\
\hline & & Amygdalin & -4.9 \\
\hline & & Shionoside C & -4.7 \\
\hline & & Protostemonine & -4.7 \\
\hline & & Norephedrine & -4.2 \\
\hline & & Praeruptorin A & -4.0 \\
\hline & & Praeruptorin C & -4.0 \\
\hline 6 & $S R C(4 M X Y)$ & Hyperoside & -8.6 \\
\hline
\end{tabular}

Table 3 (continued)
Table 3 (continued)

\begin{tabular}{|c|c|c|c|}
\hline No. & Target (PDB-ID) & Molecule name & $\begin{array}{l}\text { Docking score } \\
\text { (kcal/moL) }\end{array}$ \\
\hline \multirow{8}{*}{7} & \multirow{8}{*}{ PIK3R1 (5XXG) } & Kaempferol & -8.0 \\
\hline & & Praeruptorin C & -8.0 \\
\hline & & Isorhamnetin & -7.9 \\
\hline & & Amygdalin & -7.6 \\
\hline & & Kaempferol & -8.5 \\
\hline & & Quercitrin & -8.5 \\
\hline & & Isorhamnetin & -8.2 \\
\hline & & Hyperoside & -8.2 \\
\hline 8 & JUN (5FD2) & Kaempferol & -8.3 \\
\hline \multicolumn{4}{|c|}{$\begin{array}{l}\text { MAPK14, mitogen-activated protein kinase 14; PIK3CA, } \\
\text { phosphatidylinositol-4,5-bisphosphate 3-kinase catalytic } \\
\text { subunit alpha; AKT1, AKT serine/threonine kinase 1; MAPK1, } \\
\text { mitogen-activated protein kinase 1; EGFR, epidermal growth } \\
\text { factor receptor; SRC, proto-oncogene C-Src; PIK3R1, } \\
\text { phosphoinositide-3-kinase regulatory subunit } 1 \text {; JUN, } \\
\text { proto-oncogene c-Jun. }\end{array}$} \\
\hline
\end{tabular}

(PGE2) by blocking NF-kB activation. These results suggest that the platycodin saponin compounds have potential anti-inflammatory pharmacological effects (13). The data shown above suggest that the pharmacological actions of these components in ETK for cough therapy are plausible. Nevertheless, further basic pharmacological or clinical experimental validations are needed.

\section{Potential therapeutic mechanism}

In terms of potential therapeutic targets, according to descending order the degree of the PPI network and the core components-targets-pathways network, and then taking into account the top ranking targets of both, which were $\mathcal{H} U N$, PIK3CA, PIK3R1, MAPK14, EGFR, SRC, $A K T 1$, and $M A P K 1$. In addition, through literature search we can find that: by inhibiting the phosphorylation of c-fun N-terminal kinases, extracellular signal-regulated kinases, and p38, JUN inhibitors can effectively prevent the downstream activation of the mitogen-activated protein kinase signaling pathway and thus play a significant role in the treatment of inflammatory diseases such as asthma (20). Inhibitors of PIK3CA and PIK3R1 could exert antiinflammatory effects and treat cough (21). Inhibitors 
A

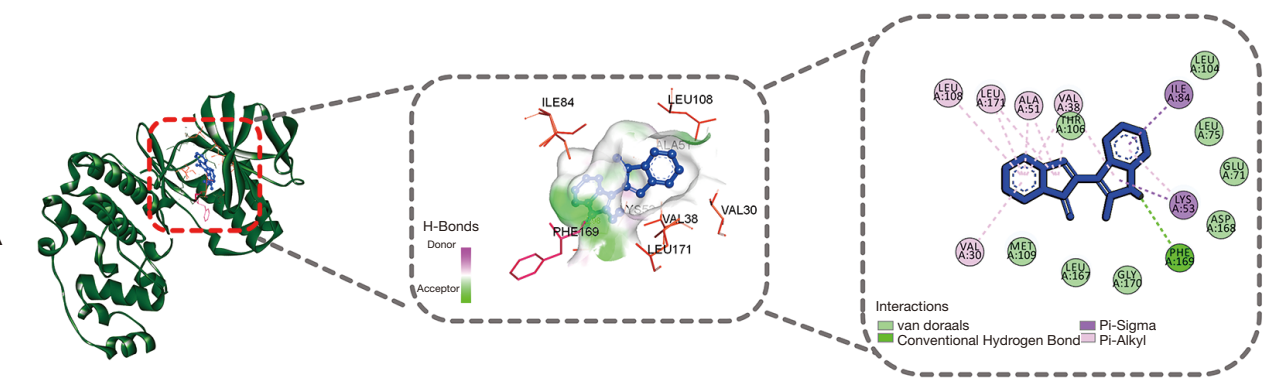

B
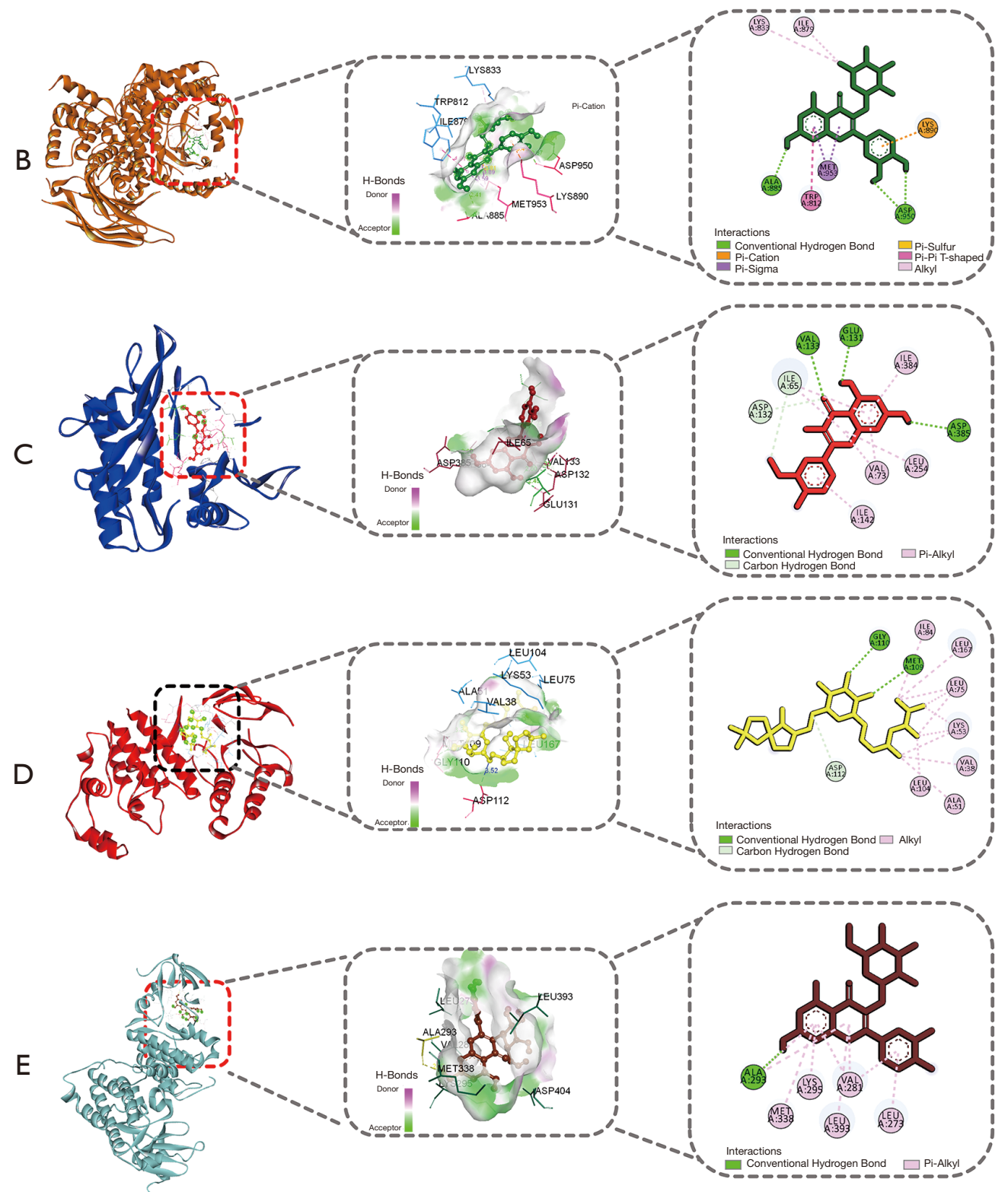

Figure 6 The molecular docking findings for the action mechanisms of active drugs on 5 target proteins. (A) Action method of indirubin (MOL001782) with target MAPK14 (PDB ID: 5OMG); (B) Action method of quercitrin (MOL000701) with target PIK3CA (PDB ID: 6AUD); (C) Action method of isorhamnetin (MOL000354) with target AKT1 (PDB ID: 6M89); (D) Action method of shionoside C (MOL010478) with target MAPK1 (PDB ID: 5OMH); (E) Action method of hyperoside (MOL004368) with target SRC (PDB ID: 4MXY). 
alleviate acute lung injury in septic mice by targeting and inhibiting MAKT14 expression (22). EGFR activators play an essential role in maintaining human bronchial epithelial cell homeostasis (23). Inhibitors of SRC blocked c-Src activation, decreased macrophage influx, and prevented airway inflammation and lung tissue destruction (24). The use of AKT1 and MAKT1 inhibitors has been shown to inhibit the production of pro-inflammatory cytokines $(I L-1 \beta$ and $I L-6)$ in LPS-stimulated mouse peritoneal macrophages, and these effects are mediated by inhibition of the activity of $I K K / I \kappa B / N F-\kappa B$, as well as phosphorylation of $A k t, E R K 1 / 2$, and $7 N K(25)$. The 8 core target proteins were docked with the potential active ingredients based on molecular docking technology. The molecular docking results of ETK were found to be satisfactory, and all had a solid binding ability, which suggested the reliability of the network pharmacological prediction results.

By analyzing GO enrichment, the mechanism of action of ETK in treating cough may be described below. The potential active ingredients are absorbed and metabolized in the body and are distributed in different regions of the cell such as organelles, cell membranes, signal synapses, and extracellular areas, and then they specifically bind to the corresponding related proteins and protein kinases in the signaling pathway. Thus, biological processes such as biological regulation, response to stimulation, metabolic response, immune system response, and signal transduction are induced, which further play a pharmacological role in inhibiting inflammation, relieving cough, and reducing phlegm. Furthermore, these putative target genes were entered into the DAVID database for KEGG pathway analysis, and a total of 20 important pathways that may be controlled by ETK in the treatment of cough were discovered. The results showed that the main signaling pathways involved in ETK treatment of cough included the biological system, immune system, signal transduction, and cellular processes, among others. Related proteins and small molecule products in different signaling pathways directly or indirectly regulate the occurrence of therapeutic effects and produce complex interactions. The reason for this is that these KEGG signaling pathways are not entirely independent but are generally related.

cAMP is a prevalent and ubiquitous second messenger in the cAMP signaling pathway. It is formed when adenylyl cyclase (AC) is activated in response to ligand binding to $\mathrm{G}$ protein-coupled receptors (GPCRs) by hormones, neurotransmitters, and other signaling molecules. cAMP is involved in a variety of important physiological activities, including metabolism, secretion, calcium homeostasis, muscular contraction, cell fate determination, and gene transcription. Research shows that exchange protein directly activated by cAMP (Epac) has a protective effect on asthmatic airway inflammation and airway remodeling (26). When inflammatory mediators are regulated in TRP channels, TRPA1 and TRPV1 channels act as critical regulators of tussive responses produced by endogenous and external agents. TRPV1 and TRPA1 antagonists reduced the tussive response to PGE2 and bradykinin (BK) in a guinea pig conscious cough model, with complete suppression achieved when both antagonists were used concurrently (27). In the calcium signaling pathway, relaxation induced by second messengers or modulators results in a localized $\mathrm{Ca}^{2+}$ response at the cell membrane, which opens large-conductance $\mathrm{Ca}^{2+}$-activated $\mathrm{K}^{+}$(BKCa) channels, resulting in hyperpolarization of the airway smooth muscle membrane, which reduced airway obstruction in a mouse model of asthma (28). Regulating the PI3K/AKT and Ras/MAPK pathways have been demonstrated to alleviate Pseudomonas aeruginosainduced acute pulmonary inflammation (29).

In summary, based on the integrity and systematicness of network pharmacology, the emphasis on drug interactions and the basic consistency with traditional Chinese medicine, this paper screened out the potential pharmacodynamic components in the ETK for the treatment of cough. In addition, when constructing and drawing the Figure $5 \mathrm{~A}$, we found that most of the potential active ingredients derived from the main components of TCM, with cough expectorant pharmacological activity characteristics. Through GO and KEGG enrichment analysis, we described the functional classification of potential therapeutic genes and signaling pathways in vivo, and then revealed the potential mechanism of ETK granules in the treatment of cough. These core signaling pathways are strongly related to the treatment of cough and have potential pharmacological effects such as antitussive, bronchiectasis, anti-inflammatory, and bacteriostasis effects. Additionally, the potential targets of ETK components can be enriched in these core signaling pathways, and each active ingredient has an effect on a minimum of one target gene, with the majority of targets acting on at least one pathway, which indirectly verifies the reliability and authenticity of network pharmacological prediction and molecular docking analysis results. However, our research has many limitations. First, our findings must be confirmed experimentally. Second, a complete database of TCM target genes was required, which improved the 
reliability of network pharmacology analysis. Third, even when network pharmacology and molecular docking data were integrated, the precise therapeutic mechanism of ETK remained unknown. Thorough knowledge of ETK and cough is contingent on the anticipated growth of many disciplines.

\section{Conclusions}

We discovered 242 active components of ETK and gathered 1,173 targets in addition to 4,400 disease-related targets for cough therapy. Additionally, 600 potential targets and 39 signaling pathways were identified. Furthermore, we have screened out tuberostemonone, quercetin, kaempferol, praeruptorin $\mathrm{E}$, stigmasterol, oroxylin $\mathrm{A}$, and other potentially active ingredients. At the same time, 8 core targets, including $\mathcal{F U N}$, PIK3CA, PIK3R1, MAPK14, EGFR, SRC, $A K T 1$, and $M A P K 1$, and 20 key pathways, including the cAMP signaling pathway, calcium signaling pathway, and PI3K-Akt signaling pathway among others, were also selected. These discoveries may pave the way for the discovery of novel chemicals and the development of new cough medications. However, further trials are required to make the findings public.

\section{Acknowledgments}

Funding: This work was supported by Key Specialized research and Development Breakthrough in Henan Province (202102310184), Henan Province Traditional Chinese Medicine Top-notch Talents Training Project [No. Yuzhong Medical Science and Education (2018) 35].

\section{Footnote}

Reporting Checklist: The authors have completed the STREGA reporting checklist. Available at https://dx.doi. org/10.21037/apm-21-2807

Conflicts of Interest: All authors have completed the ICMJE uniform disclosure form (available at https://dx.doi. org/10.21037/apm-21-2807). JH is from Yangtze River Pharmaceutical Group. The other authors have no conflicts of interest to declare.

Ethical Statement: The authors are accountable for all aspects of the work in ensuring that questions related to the accuracy or integrity of any part of the work are appropriately investigated and resolved. The study was conducted in accordance with the Declaration of Helsinki (as revised in 2013).

Open Access Statement: This is an Open Access article distributed in accordance with the Creative Commons Attribution-NonCommercial-NoDerivs 4.0 International License (CC BY-NC-ND 4.0), which permits the noncommercial replication and distribution of the article with the strict proviso that no changes or edits are made and the original work is properly cited (including links to both the formal publication through the relevant DOI and the license). See: https://creativecommons.org/licenses/by-nc-nd/4.0/.

\section{References}

1. Ding H, Shi C, Xu X, et al. Drug-induced chronic cough and the possible mechanism of action. Ann Palliat Med 2020;9:3562-70.

2. Lu X, Wang YY, Xu KK. Quality standard for ertongke granules. Anhui Med Pharm 2011;15:12.

3. Ru J, Li P, Wang J, et al. TCMSP: a database of systems pharmacology for drug discovery from herbal medicines. J Cheminform 2014;6:13.

4. Guo W, Huang J, Wang N, et al. Integrating Network Pharmacology and Pharmacological Evaluation for Deciphering the Action Mechanism of Herbal Formula Zuojin Pill in Suppressing Hepatocellular Carcinoma. Front Pharmacol 2019;10:1185.

5. Gfeller D, Grosdidier A, Wirth M, et al. Swiss TargetPrediction: a web server for target prediction of bioactive small molecules. Nucleic Acids Res 2014;42:W32-8.

6. Stelzer G, Dalah I, Stein TI, et al. In-silico human genomics with GeneCards. Hum Genomics 2011;5:709-17.

7. Szklarczyk D, Gable AL, Lyon D, et al. STRING v11: protein-protein association networks with increased coverage, supporting functional discovery in genomewide experimental datasets. Nucleic Acids Res 2019;47:D607-13.

8. Kohl M, Wiese S, Warscheid B. Cytoscape: software for visualization and analysis of biological networks. Methods Mol Biol 2011;696:291-303.

9. Jiao X, Sherman BT, Huang da W, et al. DAVID-WS: a stateful web service to facilitate gene/protein list analysis. Bioinformatics 2012;28:1805-6.

10. Trott $\mathrm{O}$, Olson AJ. AutoDock Vina: improving the speed and accuracy of docking with a new scoring function, 
efficient optimization, and multithreading. J Comput Chem 2010;31:455-61.

11. Liu Y, Shen Y, Teng L, et al. The traditional uses, phytochemistry, and pharmacology of Stemona species: A review. J Ethnopharmacol 2021;265:113112.

12. Song $\mathrm{Y}, \mathrm{Wu} \mathrm{Y}, \mathrm{Li} X$, et al. Protostemonine attenuates alternatively activated macrophage and DRAinduced asthmatic inflammation. Biochem Pharmacol 2018;155:198-206.

13. Ahn KS, Noh EJ, Zhao HL, et al. Inhibition of inducible nitric oxide synthase and cyclooxygenase II by Platycodon grandiflorum saponins via suppression of nuclear factor-kappaB activation in RAW 264.7 cells. Life Sci 2005;76:2315-28.

14. He XY, Wu LJ, Wang WX, et al. Amygdalin A pharmacological and toxicological review. J Ethnopharmacol 2020;254:112717.

15. Carr BC. Efficacy, abuse, and toxicity of over-the-counter cough and cold medicines in the pediatric population. Curr Opin Pediatr 2006;18:184-8.

16. Wei P, Huo HL, Ma QH, et al. Pharmacokinetic comparisons of five ephedrine alkaloids following oral administration of four different Mahuang-Guizhi herbpair aqueous extracts ratios in rats. J Ethnopharmacol 2014;155:642-8.

17. Li X, Zhang J, Gao W, et al. Study on chemical composition, anti-inflammatory and anti-microbial activities of extracts from Chinese pear fruit (Pyrus bretschneideri Rehd.). Food Chem Toxicol 2012;50:3673-9.

18. Ye P, Yang XL, Chen X, et al. Hyperoside attenuates OVAinduced allergic airway inflammation by activating $\mathrm{Nrf} 2$. Int Immunopharmacol 2017;44:168-73.

19. Yu P, Cheng S, Xiang J, et al. Expectorant, antitussive, anti-inflammatory activities and compositional analysis of Aster tataricus. J Ethnopharmacol 2015;164:328-33.

20. Su XD, Jang HJ, Li HX, et al. Identification of potential inflammatory inhibitors from Aster tataricus. Bioorg

Cite this article as: Chen $\mathrm{YL}, \mathrm{Li}$ WX, Zhang H, Wang XY, Zhang SQ, Zhang ML, Han J, Li K, Feng KR, Chen XF, Tang JF. Study on the mechanism of ErtongKe granules in the treatment of cough using network pharmacology and molecular docking technology. Ann Palliat Med 2021;10(11):11415-11429. doi: 10.21037/apm-21-2807
Chem 2019;92:103208.

21. Norman P. Evaluation of WO2013117503 and WO2013117504: the use of PI3K inhibitors to treat cough or idiopathic pulmonary fibrosis. Expert Opin Ther Pat 2014;24:719-22.

22. Ding L, Gao X, Yu S, et al. miR-128-3p enhances the protective effect of dexmedetomidine on acute lung injury in septic mice by targeted inhibition of MAPK14. J Bioenerg Biomembr 2020;52:237-45.

23. Yamaoka T, Arata S, Homma M, et al. Blockade of EGFR Activation Promotes TNF-Induced Lung Epithelial Cell Apoptosis and Pulmonary Injury. Int J Mol Sci 2019;20:4021.

24. Geraghty P, Hardigan A, Foronjy RF. Cigarette smoke activates the proto-oncogene c-src to promote airway inflammation and lung tissue destruction. Am J Respir Cell Mol Biol 2014;50:559-70.

25. Park SY, Park GY, Ko WS, et al. Dichroa febrifuga Lour. inhibits the production of IL-1beta and IL-6 through blocking NF-kappaB, MAPK and Akt activation in macrophages. J Ethnopharmacol 2009;125:246-51.

26. Chen YF, Huang G, Wang YM, et al. Exchange protein directly activated by cAMP (Epac) protects against airway inflammation and airway remodeling in asthmatic mice. Respir Res 2019;20:285.

27. Grace M, Birrell MA, Dubuis E, et al. Transient receptor potential channels mediate the tussive response to prostaglandin E2 and bradykinin. Thorax 2012;67:891-900.

28. Deshpande DA, Wang WC, McIlmoyle EL, et al. Bitter taste receptors on airway smooth muscle bronchodilate by localized calcium signaling and reverse obstruction. Nat Med 2010;16:1299-304.

29. Hou Y, Nie Y, Cheng B, et al. Qingfei Xiaoyan Wan, a traditional Chinese medicine formula, ameliorates Pseudomonas aeruginosa-induced acute lung inflammation by regulation of PI3K/AKT and Ras/MAPK pathways. Acta Pharm Sin B 2016;6:212-21. 